\title{
Improving Accuracy and Efficiency of Mutual Information for Multi-modal Retinal Image Registration using Adaptive Probability Density Estimation
}

\author{
P. A. Legg ${ }^{1,2}$, P. L. Rosin ${ }^{1}$, D. Marshall ${ }^{1}$ and J. E. Morgan ${ }^{3}$ \\ phil.legg@cs.ox.ac.uk, paul.rosin@cs.cf.ac.uk, \\ dave.marshall@cs.cf.ac.uk,morganje3@cf.ac.uk \\ ${ }^{1}$ School of Computer Science, Cardiff University, UK. \\ ${ }^{2}$ Department of Computer Science, University of Oxford, UK. \\ ${ }^{3}$ School of Vision Sciences and Optometry, Cardiff University, UK.
}

\begin{abstract}
Mutual Information (MI) is a popular similarity measure for performing image registration between different modalities. MI makes a statistical comparison between two images by computing the entropy from the probability distribution of the data. Therefore, to obtain an accurate registration it is important to have an accurate estimation of the true underlying probability distribution. Within the statistics literature, many methods have been proposed for finding the 'optimal' probability density, with the aim of improving the estimation by means of optimal histogram bin size selection. This provokes the common question of how many bins should actually be used when constructing a histogram. There is no definitive answer to this. This question itself has received little attention in the MI literature, and yet this issue is critical to the effectiveness of the algorithm. The purpose of this paper is to highlight this fundamental element of the MI algorithm. We present a comprehensive study that introduces methods from statistics literature and incorporates these for image registration. We demonstrate this work for registration of multi-modal retinal images: colour fundus photographs and scanning laser ophthalmoscope images. The registration of these modalities offers significant enhancement to early glaucoma detection, however traditional registration techniques fail to perform sufficiently well. We find that adaptive probability density estimation heavily impacts on registration accuracy and runtime, improving over traditional binning techniques.
\end{abstract}


Keywords: Mutual information; image registration; probability estimation; histogramming.

\section{Introduction}

Image registration is the task of finding the spatial transformation that gives correct matching correspondence between two images. Registration is widely used in many application areas. In particular, registration of images from different modalities has become increasingly common in areas such as Medical Imaging for in-depth patient analysis. However, the difficulty is that by their very nature, multi-modal image pairs may have no clearly defined relation between corresponding image intensities.

Mutual Information (MI) has become a popular similarity measure for multi-modal registration. The algorithm was simultaneously proposed by both Viola [1] and Collignon [2], and since then has stimulated much interest. Derived from Information Theory, MI is based on statistical comparison between the two images being registered. This differs from traditional registration techniques that rely on direct pixel intensity calculation such as Normalized Cross-Correlation. Given the floating image $A$ and the corresponding area from the reference image $B$, MI can be defined as:

$$
M I(A, B)=H(A)+H(B)-H(A, B)
$$

where $H(A)$ is the entropy of image $A, H(B)$ is the entropy of image $B$ and $H(A, B)$ is the joint entropy of both $A$ and $B$. The transformation that maximizes $M I(A, B)$ should result in the correct registration. Studholme [3] extended this by introducing Normalized MI that is designed to handle partial overlap of the images. This is defined as:

$$
N M I(A, B)=\frac{H(A)+H(B)}{H(A, B)} \text {. }
$$

Entropy gives a measure of the amount of information that a given signal may contain, and forms the basis of MI. For a signal $X$ consisting of $n$ elements, Shannon's entropy [4] is defined as:

$$
H(X)=-\sum_{i=0}^{n} p(i) \log _{2} p(i)
$$




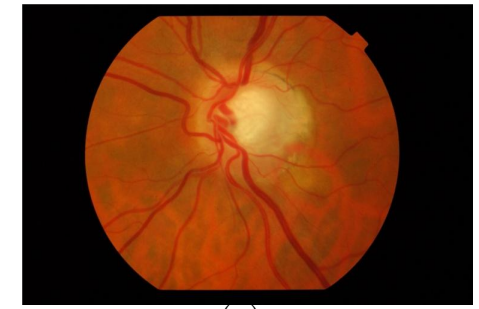

(a)

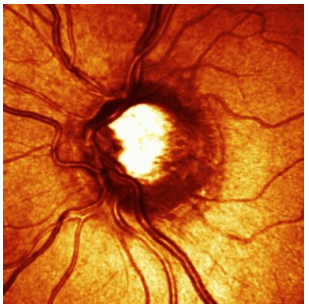

(b)

Figure 1: (a) Fundus colour photograph. (b) Scanning laser ophthalmoscope image.

where $p(i)$ is the probability of value $i$ occurring within the data set. The amount of information for a given value is inversely related to its probability, meaning that if the probability of a particular value occurring is low then this returns a greater amount of information than if the probability of the value is high. It can be thought of that the more rare the occurrence of an event, the more important it is when that event does occur. For an image, if there are many identical intensity values (such as background) then the entropy result will be low. However, an image that has lots of detail will return a much larger entropy, due to having a greater variety of intensity values. From this description, entropy can also be thought of as a measure for the dispersion of the probability.

This can be extended further to compute the entropy of two signals, known as the joint entropy. The joint entropy defines the amount of information given by the combination of both signals. Using Shannon's entropy, we can define joint entropy of two signals $A$ and $B$, consisting of $n$ and $m$ elements respectively, as:

$$
H(A, B)=-\sum_{i=0}^{n} \sum_{j=0}^{m} p(i, j) \log _{2} p(i, j)
$$

where $p(i, j)$ is the probability of value $i$ occurring in $A$ at the same time as $j$ occurs within $B$. From this, it can be seen that it is the probability distribution of the two images being registered that forms the basis of the MI algorithm. Therefore how the probability distribution is actually estimated could have a significant impact on the performance of the MI algorithm.

For this study we are particularly interested in the registration of a challenging dataset comprised of multi-modal retinal image data. The two 
modalities that are to be registered together are colour fundus photographs and confocal scanning laser ophthalmoscope (SLO) images (Figure 1). Both modalities capture high quality images from the eye of the optic nerve head $(\mathrm{ONH})$, with the fundus photograph recording the clinical appearance and the SLO image providing quantitative information such as the retinal surface reflectivity and topographic structure [5]. Whilst it is apparent that similar features exist in both images it is also very clear that there are significant differences in how these are represented. Fusion of the two images would bring together complementary information and improve $\mathrm{ONH}$ analysis for the early detection of glaucoma.

In this paper we conduct a comprehensive study that shows how histogram bin size estimation methods can heavily impact on MI registration results. We present a number of histogram bin size methods from statistics literature (Section 3). We also present alternative probability estimation methods that have also become popular in the literature (Section 4). Using these methods we carry out extensive testing of MI registration (Section 5). Finally, we provide discussion on the study (Section 6).

\section{Literature Review}

MI relies on a number of factors that need to be carefully considered in order to perform accurate image registration. Pluim [6] gives a thorough survey of the algorithm along with an overview of influencing factors that affect registration performance. Beirlant et al. [7] provides a mathematical overview of entropy estimation methods and discusses the associated parameters. Paninski [8] also looks at the estimation of entropy methods, and extends this to consider the estimation of MI. The report by Egnal [9] introduces the topic of probability estimation in relation to MI well, and also introduces the notion of histogramming in relation to MI.

Birgé addresses the issue of histogramming in his comprehensive study [10] that sets out to answer the question, "How many bins should be put in a regular histogram?". Whilst the histogram remains perhaps the most simple to understand method of probability estimation, there is still no definitive answer to this question. Instead, many previous works tend to use a value that seems appropriate, with no statistical justification behind this. Certainly in the MI literature the issue of how many bins to use in the histogram is quite often over-looked. Many studies seem to take the approach of populating the histogram by binning equal intensity values together [6]. For 8-bit images, 
the number of bins would cover the full range of 256 gray-levels to maintain intensity independence, although not necessarily stated. Collignon et al. [2] do not initially specify bin size, however later work by Maes et al. [11] states that they use 256 bins. They also mention that they do not investigate the influence that bin size may have.

More recent works have began to realise the importance of histogram bin size, however they typically rely on experimental selection rather than on any statistical basis. Dowson and Bowden [12] make the point that MI is not invariant to the bin size, although do not demonstrate the effects of altering this parameter. Lachner states the importance of determining correct bin size based on the trade-off between histogram variance and bias [13], concluding that 64 bins provide satisfactory results for their experiments. In contrast to this, Ritter et al. [14] performs registration of intra-modal fundus photographs using just 4 histogram bins, again determined through experimental testing. Nam et al. [15] experiment using 5, 10 and 32 bins, concluding that 10 bins gives the best results for their data. Similarly, Kang et al. [16] assessed using 4, 8, 16, 32 and 64 bins, concluding that the best number of bins is between 4 and 16. It is often suggested, as in [17], to simply use a power of 2 as a suitable value for bin size, or as in [18], 'a low number of bins'.

However, in the statistics literature there has actually been much work carried out on selecting the optimal number of bins for histogram construction, with both Birgé [10] and Davies [19] giving comprehensive surveys on the topic. The earliest work dates back to 1926, when Herbert Sturges proposed a rule to calculate the number of bins based on properties of the data being organized [20]. Egnal [9] and Lachner [13] actually refer to Sturges' Rule when discussing MI, however do not draw any conclusions as to whether this is beneficial to the algorithm. After the introduction of Sturges' Rule, it was not until 1979 that perhaps the second most recognized bin size rule was proposed - Scott's Rule [21]. It had been suggested that Sturges' Rule over-smoothed the histogram and so a better rule was necessary [22]. From these two rules came many other variations, such as the Freedman-Diaconis Rule [23], Devroye's Rule [24], Taylor's Rule [25] and Doane's Rule [26]. Further modifications on existing works are also proposed such as Scott's Rule with a skewness factor [27] and Sturges' Rule with the kurtosis of the histogram [28]. The impact of bin size has also be studied for other applications, such as by Shimazaki and Shinomoto [29] for the construction of time histograms. 
From our review of the literature, it is evident that bin size selection has not been fully explored within MI. However, probability distribution provides the fundamental basis of the algorithm and could dramatically affect the registration performance.

\section{Histogram Bin Size Selection}

When a continuous analogue signal such as an image is discretized for the purpose of digital processing, artefacts occur due to intensity and spatial quantization. Probability density estimation is the task of predicting the shape of the true distribution based on the sampled data set. By finding the 'optimal' probability density representation it may be possible to obtain an estimate close to that of the original signal. Similarly, by adapting the probability density representation further, distracting artefacts such as noise may be reduced to leave only salient features in the image.

The simplest and most common approach to probability density estimation is by use of a histogram. Typically, an image histogram shows how many occurrences there are of each independent intensity value within the data set. Each set of occurrences is a 'bin' within the histogram. We can also reduce the number of bins to group together intensities. By grouping intensities, we can reduce the number of empty bins that occur within the distribution (since an image will unlikely consist off all possible intensity values). However, reducing the number of bins too far will degrade the information in the image dramatically meaning that distinct features are lost. Histograms tend to take two forms; regular and irregular. When discussing the number of bins for a histogram it is typical to use a regular histogram where bin width is uniform throughout. Irregular histograms, whilst they can provide greater flexibility in classification, would most likely require some intervention by the user which makes this unsuitable for an automated registration procedure.

In Section 2 we have shown that there are many statistical methods for computing bin size selection, although these are not commonly adopted in Computer Vision applications. Unfortunately, of all these methods, there is no single approach that is universally recognized as the best approach for bin size selection. This is because each method relies on assumptions of the underlying data, i.e. what model is chosen to fit that distribution. Typically, how well a model can be fit to the data is measured by a loss function that is to be minimized. The Hellinger Distance and $L_{n}$-norms are two common loss functions that may be used to evaluate the construction of a regular 
histogram. For a review of loss functions in application to regular histograms, see Birgé and Rozenholc [10]. It is the combination of the different possible distribution models and the different possible loss functions that leads to the large number of methods in the statistics literature for selecting the possible number of bins.

For our study, we are constructing the histogram for the purpose of further processing, and so we are not concerned with whether the distribution can improve a particular loss function as such. Instead, we assess each method by how well it can improve registration. We wish to minimise the registration error by adopting different bin size selection methods. The method that results in the least error can be regarded as the most suitable bin size selection method for registration.

\subsection{Sturges' Rule}

Sturges' Rule [20] was originally proposed in 1926 and is still commonly used today in many statistical computer packages. The rule provides a simple formula that is based on properties from the data being classified in the histogram. Sturges' Rule makes the assumption that a histogram consisting of Normal data can be approximated by a binomial distribution. The rule defines the bin width as $w=1+\log _{2}(n)$ where $n$ is the number of elements within the data set. We can then simply determine the number of bins to be $r / w$ where $r$ is the range of the data set. Since Sturges' Rule assumes that the data is normally distributed it could then give inaccurate results where this does not hold true.

Figure 2 shows an example of applying Sturges' Rule to the fundus photograph and the SLO image. Both of these images are $259 \times 266$ pixels, meaning that there are 68096 pixels in each image. By applying Sturges' Rule, it can be seen that the number of bins in the histogram is reduced significantly. For the original fundus image there are 204 occupied bins (i.e. unique intensity values in the image), which can be reduced to just 19 bins after applying Sturges' Rule. Likewise, for the SLO image this reduction is from 246 to just 19. The number of pixels and the data range are the two main factors in Sturges' Rule.

There are two benefits that the new histogram can offer. Firstly, the original histogram consists of frequently unpopulated bins, which are likely an artefact created by the discretization of the data during image acquisition. By grouping intensities together we can eliminate such artefacts and populate all bins in the distribution. Secondly, entropy is computed as a summation 


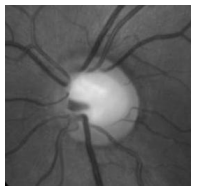

(a)

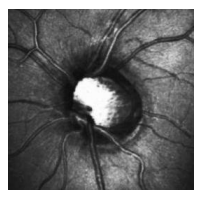

(d)

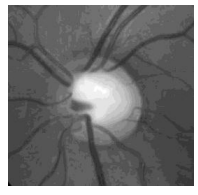

(b)

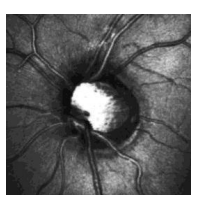

(e)

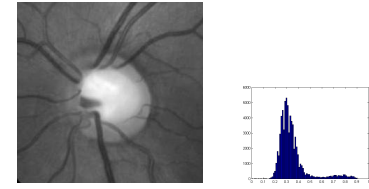

(c)

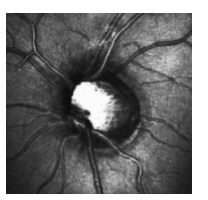

(f)

Figure 2: Fundus image and associated histogram (a) original, (b) computed using Sturges' Rule (19 bins), (c) computed using Scott's Rule (84 bins). SLO image and associated histogram (d) original, (e) computed using Sturges' Rule (19 bins), (f) computed using Scott's Rule (62 bins). Image representations are modified based on the number of histogram bins.

of all probabilities in the data set, which means that if there are less unique intensity values then less calculation is required leading to improved runtime.

It is also important to see how the reduced intensity images replicate the originals. It can be seen that the fundus image has lost much detail in the background and created a 'patch-like' effect of intensity regions, yet the key features such as the blood vessels and the optic disc still appear very clear. This reduction of intensities could be regarded as 'cleaning up' the image, by eliminating noisy artefacts such as the background that could quite easily mislead the registration. In the case of the SLO, whilst there is a similar reduction to the number of unique intensities, the appearance does not look particularly different. In this situation, the process manages to preserve much of the original detail although we still benefit from the fact that the number of intensities is heavily reduced.

\subsection{Scott's Rule}

Scott's Rule [21] is similar to Sturges' Rule, however is based on the standard deviation of the data. Given two different images that have the same intensity range and same size, Sturges' Rule would give identical bin size whereas Scott's Rule would give a bin size based on the actual intensity values being considered. Scott's Rule defines the bin width as $3.49 \sigma n^{-1 / 3}$, where $\sigma$ is the standard deviation of the data and $n$ is the number of elements 
within the set. As with Sturges' Rule, this assumes that the data is Normal.

Figure 2 also shows the affect of applying Scott's Rule to the images. Whereas Sturges' Rule reduced the number of unique intensity values to just 19, Scott's Rule suggests that 84 and 62 bins should be used for the fundus photograph and the SLO image respectively. Comparing the histograms, it is clear that Sturges' Rule reduces the number of intensities greatly and so the histogram now consists of larger steps between each intensity bin. However, Scott's Rule reduces the bin size whilst maintaining a relatively small step size between each intensity bin in the histogram. For the SLO image, the histogram representation using Scott's Rule is very similar in shape to that of the original image. This highlights the trade-off between using low number of bins whilst trying to preserve the original probability density estimation.

\subsection{Variations based on Scott's Rule and Sturges' Rule}

The introduction of Scott's Rule gave birth to many variations on the rule that could be used for bin size selection. Taylor [25] and Kanazawa [30] give the bin width as $2.29 \sigma^{2 / 3} n^{-1 / 3}$, whilst Devroye and Györfi [24] give the bin width as $2.72 \sigma n^{-1 / 3}$. Freedman and Diaconis also took a similar approach that is described as more robust to Scott's Rule [31], using the interquartile range (IQR) of the data, which gives the bin width as $2(I Q R) n^{-1 / 3}$.

As mentioned previously, both Sturges' Rule and Scott's Rule (and its variants) assume that the data consists of a Normal distribution. In Figure 2 it can be seen that this is not the case as both histograms are skewed. Since it is known that the data does not fit the assumptions of the model, the obtained results are likely to be sub-optimal. Typically, it is thought that these methods suggest too few bins (or rather, the equivalent being too large a bin width). Doane [26] proposed a method that extends Sturges' Rule to account for the skewness of the data. Given that $n$ is the number of elements, $X_{i}$ is an element in the set and $\bar{X}$ is the mean of the set, Doane proposes the number of bins as:

$$
\log _{2}(n)+1+\log _{2}\left(1+\frac{\sqrt{b}}{\sigma \sqrt{b}}\right)
$$

where

$$
\sqrt{b}=\frac{\sum_{i=1}^{n}\left(X_{i}-\bar{X}\right)^{3}}{\left[\sum_{i=1}^{n}\left(X_{i}-\bar{X}\right)^{2}\right]^{(3 / 2)}}
$$

and 


$$
\sigma \sqrt{b}=\sqrt{\frac{6(n-2)}{(n+1)(n+3)}} .
$$

Scott also proposes a method that extends his own method, where Scott's Rule is multiplied by a skewness factor [27], as defined by:

$$
\text { skewness factor }=\frac{2^{1 / 3} \sigma}{e^{5 \sigma^{2} / 4}\left(\sigma^{2}+2\right)^{1 / 3}\left(e^{\sigma^{2}}-1\right)^{1 / 2}} .
$$

By considering the extent of the skew, these approaches tend to recommend using a slightly greater number of bins for the histogram.

Similar to measuring the skewness factor, we can also consider the kurtosis of a histogram. What the kurtosis measures is how peaked or flat the distribution is in comparison to a Normal distribution. If the data has a high kurtosis then the data has a distinct steep peak close to the mean of the data. A low kurtosis shows that the data has a much flatter distribution. Kurtosis is defined as:

$$
K=\frac{\sum_{i=1}^{n}\left(X_{i}-\bar{X}\right)^{4}}{(n-1) \sigma^{4}}
$$

where $n$ is the number of elements, $\bar{X}$ is the mean of the data and $\sigma$ is the standard deviation. In Wichard [28], Sturges' Rule is adapted to include the kurtosis measure, given by:

$$
\log _{2}(n)+1+\log _{2}(1+K * \sqrt{n / 6})
$$

We have presented a number of different bin size strategies that rely of different statistics of the data. Sturges' rule simply uses the number of elements in the data, whereas Scott's rule (and its variants) make use of the standard deviation of the data. Freedman and Diaconis use the IQR of the data instead of the standard deviation. Finally, we have seen methods that introduce skewness (Doane and Scott) and kurtosis (Wichard) measures regarding the data distribution. By incorporating such statistics we develop a greater understanding of the data which can be used to create an improved model for the probability distribution. Whilst there is no formally correct solution for how the data should be binned, we are interested to see whether a particular bin size method can improve registration accuracy when used to compute MI. 


\subsection{Joint Histogram Bin Size}

In order to compute MI, we need to consider probability estimation from not only the marginal histogram, but also the combined joint histogram. There is little mention in the literature regarding joint histogram bin size selection. Most existing works tend to take the original histogram bins to a power of 2 (e.g. $256^{2}=65536$ bins). Xie [32] suggests that as a rough guide, the number of bins used for the joint histogram should give an average of at least one sample per bin. As with the standard histogram, ideally there would be no empty bins within the distribution and so this approach seems plausible, however it is not derived from any statistical justification. Moreover, such an approach is likely to greatly underestimate the number of bins for the joint histogram, resulting in much loss of data with regards to the intensity correspondence between the two images. We decide to use an $m \times n$ joint histogram, where $m$ is the number of bins to use for our floating image and $n$ is the number of bins to use for our reference image, as determined by the bin size selection methods discussed previously. In doing this, we maintain the statistical relationship with the marginal probability estimation. This approach eliminates a large number of redundant empty bins compared to when using $256^{2}$ bins, whilst also preserving enough bins to ensure that the probability estimation remains meaningful. We acknowledge that this approach is not optimal, however it does serve to draw the attention of the community to the importance of bin size in joint probability estimation. The topic of joint probability estimation remains a largely unexplored topic for MI, and is outside the scope for this current work.

\section{Alternative Methods for Probability Density Estimation}

Along with histogram bin size we also investigate into alternative probability estimation methods which we include in our experimental testing. Whilst these methods are more computationally demanding, it is thought that they give a closer representation of the original signal.

\subsection{Kernel Density Estimation}

Kernel Density Estimation [1] aims to resolve the disadvantages of a histogram, most notable being the lack of a continuous function due to its step-like fashion. The method adapts the traditional histogram by convolving the histogram with a fixed kernel [33]. By doing so, each point in the

distribution is spread over multiple bins based on the chosen kernel which 
reduces the effect of discrete bin entries. The shape of the kernel determines how the data is spread in the distribution. To show how this method relates to the histogram, supposing we chose the kernel to be a square block, with a width equal to our histogram bin width, then this would essentially be equivalent to our original histogram. However, if we choose the kernel to be a smooth Gaussian curve, this can provide a much more desired result. Similar to this is the idea of using a B-Spline curve to spread the probability distribution in the same fashion [34]. As well as the shape of the kernel, we need to ensure that the kernel size is chosen well to suit the distribution. If the kernel is too small or too large this would lead to an under-smoothed or over-smoothed distribution respectively.

\section{2. $k$-Nearest Neighbour Density Estimation}

The $k$-Nearest Neighbour $(k \mathrm{NN})$ approach [35] is a commonly used tool for classification that relies on computing the average distance between a given point and its $k$-nearest neighbours within the data set. Clearly this method is highly dependent on neighbouring data points, and also takes into account the spread of the data. Essentially, $k \mathrm{NN}$ is closely related to KDE then. In KDE, the kernel remains a fixed size and captures a variable number of samples at each data point. In $k \mathrm{NN}$, the kernel size becomes variable so as to capture a fixed number of samples as defined by $k$. As with previous methods, careful parameter selection for $k$ is important, causing an undersmoothed or over-smoothed distribution if set too low or too high. In the current literature, it is recognized that $k \mathrm{NN}$ can be very computationally expensive for large data sets [36]. This is due to computing the size of the kernel based on the neighbourhood points for every element in the data set. In the context of MI registration, this could easily become exceptionally expensive leading to an impractical system for performing fast registration.

\subsection{NP-Windows}

NP (Non-Parametric) Windowing is a method proposed by Dowson et al. [37] which aims to improve the probability distribution by effectively sampling the image at an infinite resolution. One advantage that NP-Windows has over other approaches we have seen is that it does not require any parameters to be selected beforehand. Given an intensity at point $(x, y)$ in the image, along with three neighbouring intensities at points $(x+1, y),(x, y+1)$ and $(x+1, y+1)$, rather than populating just these bins in the histogram, they create two triangles using these intensity points, and then determine all 
the intensities that occur within the triangle area. Each intensity bin is then incremented based on the area of the two triangles. This approach accounts for absent intensity variations between a pixel and its neighbour, as before, aiming to reduce the artefacts introduces by pixel discretization. Rajwade proposed a similar idea [38] that interpolates the image to an infinite resolution. This approach could be seen as histogram interpolation, whereby we could scale the histogram by a given factor and then scale back to the original size. The scale factor would affect how much smoothing is introduced into the histogram, but also lead to expensive computation to perform. Compared to standard histogram binning this method is very slow to compute, which due to computational demands of the algorithm is not surprising. We note that the authors' work suggests using GPU optimization to compute NP-Windows in a satisfactory time, however this is beyond the scope of our study.

\section{Evaluation of Probability Density Estimation Methods}

The data set used consists of 135 matching image pairs captured from the human eye. For each pair, there is a colour fundus photograph and an SLO image. The original resolution of each fundus photograph is $3008 \times 1960$. The SLO images are captured using the Heidelberg Retinal Tomograph II (HRT II) [39] device. The field of view for each SLO image is $15 \times 15$ degrees and the original resolution is $384 \times 384$. The data set consists of both left and right eyes and shows various stages of the glaucoma disease ranging from no sign of infection to highly glaucomatous. Whilst all the SLO images are of a high clinical standard, there are some cases where slight blurring has occured in the image. This is due to subtle movement in the eye during the acquisition process (microsaccades). The data set represents a wide variety of different scenarios that a clinician would encounter when capturing these two image modalities. All images were taken by an expert clinician, who also provided ground truth data using a manual alignment tool developed specifically for this task. For this study, we perform registration to assess the effect of probability estimation in MI and Normalized MI. In order to evaluate the registration accuracy we will consider a variety of different implementations.

Registration can be thought of in two parts: the transformation search that maps the floating image to the reference image and the similarity measure that makes the comparison between the overlap of the two images (which in this study is MI). How the transformation search is performed can great 
impact both the registration accuracy and runtime efficiency. A naive approach would use an exhaustive search that considers all possible transformations. Even for just rigid transforms only (rotation and translation) this is far too time-consuming and not practical. Instead, an optimized search scheme is required. In our testing we shall consider two well-known optimization methods; Nelder-Mead Simplex and Simulated Annealing (details and implementation for each of these can be found in [40]). Whilst these schemes can speed up the search process, it should be noted that they can also give incorrect results should the search space contain local maxima that 'trap' the search process. Therefore it is important that the search scheme works well in conjunction with the similarity measure. We choose Nelder-Mead Simplex and Simulated Annealing since these are two common optimization methods that are well-known within the community. While many alternative search optimization schemes exist, it should be recognized that the main focus of this paper is to improve the similarity measure being optimized. By improving the convergence of the similarity measure, there becomes less uncertainty in the performance of the optimization method.

Another possible technique to improve registration is the inclusion of a multi-resolution image pyramid. Registration is performed at the coarse (top) level of the pyramid which acts as the initialization point for the next level down. This is done for each level of the pyramid until reaching the fine (bottom) level which consists of the original image. This approach can dramatically improve runtime since the low resolution images can be processed quicker and are used to initialize registration of the high resolution images. Multi-resolution pyramids are also commonly used in 3D and 4D registration schemes. So whilst the original image data may have many more data points than our 2D registration example, the coarse levels of the pyramid will actually be quite similar, meaning that the same bin size strategies could be applied also.

As part of our testing we shall consider registration both with and without an image pyramid. We use a 3-level pyramid where the image is taken at full, half and quarter resolution. In each case, registration is initialized by placing the template image in the centre of the reference image. We use search optimization strategies to find the translation parameters. The rotation parameter is known to be within $\pm 3^{\circ}$ (with $0.5^{\circ}$ increments). Since the rotation parameter space is much smaller than the translation space, we choose to search all possible values within this range at the coarse pyramid level. This ensures that the correct rotation parameter is tested by the reg- 


\begin{tabular}{|l||r|r|r|r|r|r|}
\hline \multicolumn{1}{|l|}{ Method } & \multicolumn{2}{|c|}{ Mean } & \multicolumn{2}{c|}{ Median } & \multirow{2}{*}{ Regerr } & Runtime \\
\cline { 2 - 5 } & $\boldsymbol{T}$ & $\boldsymbol{R}$ & $\boldsymbol{T}$ & $\boldsymbol{R}$ & & \\
\hline 256 bins & 154.5 & 2.8 & 154.7 & 2.5 & 154.4 & 2.77 \\
128 bins & 141.7 & 2.5 & 148.4 & 2.5 & 141.8 & 2.19 \\
64 bins & 99.0 & 2.3 & 121.8 & 1.5 & 99.5 & 2.14 \\
32 bins & 64.8 & 2.1 & 46.8 & 1.5 & 65.6 & 2.13 \\
16 bins & 52.9 & 2.1 & 32.9 & 1.5 & 54.0 & 2.14 \\
8 bins & $\mathbf{4 9 . 8}$ & $\mathbf{1 . 8}$ & $\mathbf{3 1 . 1}$ & $\mathbf{1 . 5}$ & $\mathbf{5 0 . 9}$ & $\mathbf{2 . 1 5}$ \\
4 bins & 52.9 & 2.2 & 37.3 & 1.5 & 54.3 & 2.18 \\
\hline devroye & 42.5 & 1.9 & 18.4 & 1.5 & 43.7 & 2.62 \\
doane & 54.2 & 2.1 & 28.1 & 1.5 & 55.6 & 2.42 \\
fd & 42.7 & 2.1 & 22.0 & 2.0 & 44.0 & 3.72 \\
scott & 44.9 & 1.8 & 18.9 & 1.5 & 46.1 & 2.63 \\
+skewness & $\mathbf{4 1 . 7}$ & $\mathbf{1 . 9}$ & $\mathbf{1 5 . 8}$ & $\mathbf{1 . 0}$ & $\mathbf{4 3 . 2}$ & $\mathbf{5 . 7 0}$ \\
sturges & 51.5 & 2.0 & 25.6 & 1.5 & 52.6 & 2.30 \\
+kurtosis & 51.5 & 2.3 & 29.0 & 2.0 & 52.7 & 3.72 \\
taylor & 94.8 & 2.2 & 106.1 & 2.0 & 95.3 & 2.89 \\
\hline kde (9) & 64.5 & 2.2 & 40.6 & 2.0 & 65.6 & 4.51 \\
kde (17) & 56.6 & 2.1 & 26.5 & 1.5 & 57.7 & 5.11 \\
kde (33) & 54.3 & 2.1 & 29.4 & 1.5 & 55.5 & 6.19 \\
knn (8) & $\mathbf{5 0 . 6}$ & $\mathbf{1 . 9}$ & $\mathbf{2 5 . 8}$ & $\mathbf{1 . 5}$ & $\mathbf{5 1 . 8}$ & $\mathbf{1 0 2 . 7 6}$ \\
knn (16) & 51.7 & 2.0 & 26.3 & 1.5 & 52.9 & 120.09 \\
knn (32) & 51.6 & 2.0 & 25.2 & 1.5 & 52.9 & 125.45 \\
npwin & 59.2 & 2.0 & 42.2 & 1.5 & 60.1 & 84.99 \\
\hline
\end{tabular}

Table 1: Registration error results: MI combined with an image pyramid using simplex search. Table shows fixed bin size (top), adaptive bin size (middle), and alternative estimation methods (bottom). Translation and $R e g_{\text {err }}$ are given in pixels, rotation is given in degrees, and runtime is given in seconds.

istration algorithm, and aims to minimize the possible error that could be introduced by using a search optimization strategy. As the pyramid is traversed the rotation search space becomes further restricted, converging to a fixed parameter by the lowest level of the pyramid. For our testing where no image pyramid is used, the full rotation range is allowed throughout the registration.

For our study, we developed a MATLAB implementation for performing multi-modal image registration. For search optimization, we use the built-in MATLAB implementation of the Nelder-Mead simplex algorithm (fminsearch), and the implementation by Vandekerckhove of Simulated Annealing (available from MATLAB Central [41]). Experiments were conducted on a standard desktop PC machine configured with a Pentium $2.6 \mathrm{GHz}$ dualcore processor, $4 \mathrm{~GB}$ of memory, and Windows 7 operating system. The registration software developed and a subset of the image data are both available to download by request to the authors.

\subsection{Registration Error Results}

To quantify the results of our experiments, we compare the registration results to the ground truth registration results as approved by an expert clinician. We compare the mean and median translation error $T$ (measured in pixels) and rotation error $R$ (measured in degrees), along with the mean 


\begin{tabular}{|l||r|r|r|r|r|r|}
\hline \multicolumn{1}{|l||}{ Method } & \multicolumn{2}{|c|}{ Mean } & \multicolumn{2}{|c|}{ Median } & \multicolumn{1}{c|}{ Regerr } & Runtime \\
\cline { 2 - 5 } & $\boldsymbol{T}$ & $\boldsymbol{R}$ & $\boldsymbol{T}$ & $\boldsymbol{R}$ & & \\
\hline 256 bins & 142.5 & 2.8 & 154.7 & 2.5 & 142.2 & 17.40 \\
128 bins & 140.1 & 2.5 & 141.3 & 2.0 & 140.2 & 13.39 \\
64 bins & 125.7 & 2.3 & 137.5 & 2.0 & 125.9 & 12.64 \\
32 bins & 98.5 & 2.1 & 122.9 & 1.5 & 99.1 & 12.71 \\
16 bins & $\mathbf{7 8 . 9}$ & $\mathbf{1 . 9}$ & $\mathbf{8 4 . 8}$ & $\mathbf{1 . 5}$ & $\mathbf{7 9 . 5}$ & $\mathbf{1 3 . 1 2}$ \\
8 bins & 80.6 & 2.2 & 92.9 & 2.0 & 81.6 & 13.07 \\
4 bins & 92.1 & 2.5 & 97.6 & 2.5 & 92.8 & 13.28 \\
\hline devroye & 61.2 & 1.8 & 22.0 & 1.5 & 62.1 & 16.79 \\
doane & 76.1 & 2.0 & 82.5 & 1.5 & 76.9 & 15.49 \\
fd & $\mathbf{4 5 . 6}$ & $\mathbf{1 . 9}$ & $\mathbf{2 7 . 6}$ & $\mathbf{1 . 5}$ & $\mathbf{4 6 . 6}$ & $\mathbf{2 7 . 1 9}$ \\
scott & 55.9 & 1.8 & 23.0 & 1.5 & 56.9 & 16.80 \\
+skewness & 57.4 & 1.9 & 21.0 & 1.5 & 58.3 & 38.26 \\
sturges & 76.6 & 2.1 & 70.3 & 1.5 & 77.2 & 14.33 \\
+kurtosis & 77.2 & 2.1 & 83.4 & 1.5 & 78.1 & 32.32 \\
taylor & 132.6 & 2.4 & 141.5 & 2.5 & 132.7 & 20.73 \\
\hline kde (9) & 100.6 & 2.2 & 120.4 & 2.0 & 101.1 & 32.99 \\
kde (17) & 87.1 & 2.4 & 102.2 & 2.0 & 87.8 & 39.06 \\
kde (33) & 76.6 & 1.9 & 78.3 & 1.5 & 77.3 & 49.47 \\
knn (8) & 83.5 & 1.9 & 103.3 & 1.5 & 84.3 & 894.54 \\
knn (16) & 82.9 & 2.1 & 96.6 & 2.0 & 83.5 & 920.32 \\
knn (32) & 80.9 & 2.0 & 91.1 & 1.5 & 81.4 & 1099.40 \\
npwin & $\mathbf{6 9 . 5}$ & $\mathbf{2 . 3}$ & $\mathbf{6 9 . 5}$ & $\mathbf{1 . 5}$ & $\mathbf{7 0 . 4}$ & $\mathbf{7 4 2 . 1 3}$ \\
\hline
\end{tabular}

Table 2: Registration error results: MI combined with an image pyramid using simulated annealing. Table shows fixed bin size (top), adaptive bin size (middle), and alternative estimation methods (bottom). Translation and $\operatorname{Reg}_{\text {err }}$ are given in pixels, rotation is given in degrees, and runtime is given in seconds.

registration error based on the 4 corner points of the template image, defined as $R e g_{\text {err }}$ (measured in pixels). This is calculated by measuring the distance for each corner point between the registration result and the ground truth. Each experiment is carried out using the complete set of 135 retinal image pairs. Four scenarios were tested in our experiments: MI with an Image Pyramid, NMI with an Image Pyramid, MI without an Image Pyramid and NMI without an Image Pyramid. In this paper we report only the most significant results. The complete set of results can be found in [42].

\subsubsection{MI with a Multi-Resolution Image Pyramid}

Tables 1 and 2 show a great difference in transformation error between the different probability estimation methods. It can be seen that using 256 bins in the histogram gives the worst result. Whilst a typical image histogram may consist of 256 bins, it seems that this does not yield great results when there is further processing to be done such as constructing a joint histogram. This is most likely due to sparsely-populated bins within the joint histogram that can lead to a poor entropy estimate. Previous studies have suggested that a low number of bins should be used for the histogram which can be observed in the table. As the number of fixed bins reduces, the registration error is minimized. We noted earlier that no suggestion to how low this number of bins should be is given and that many papers tend to find the 


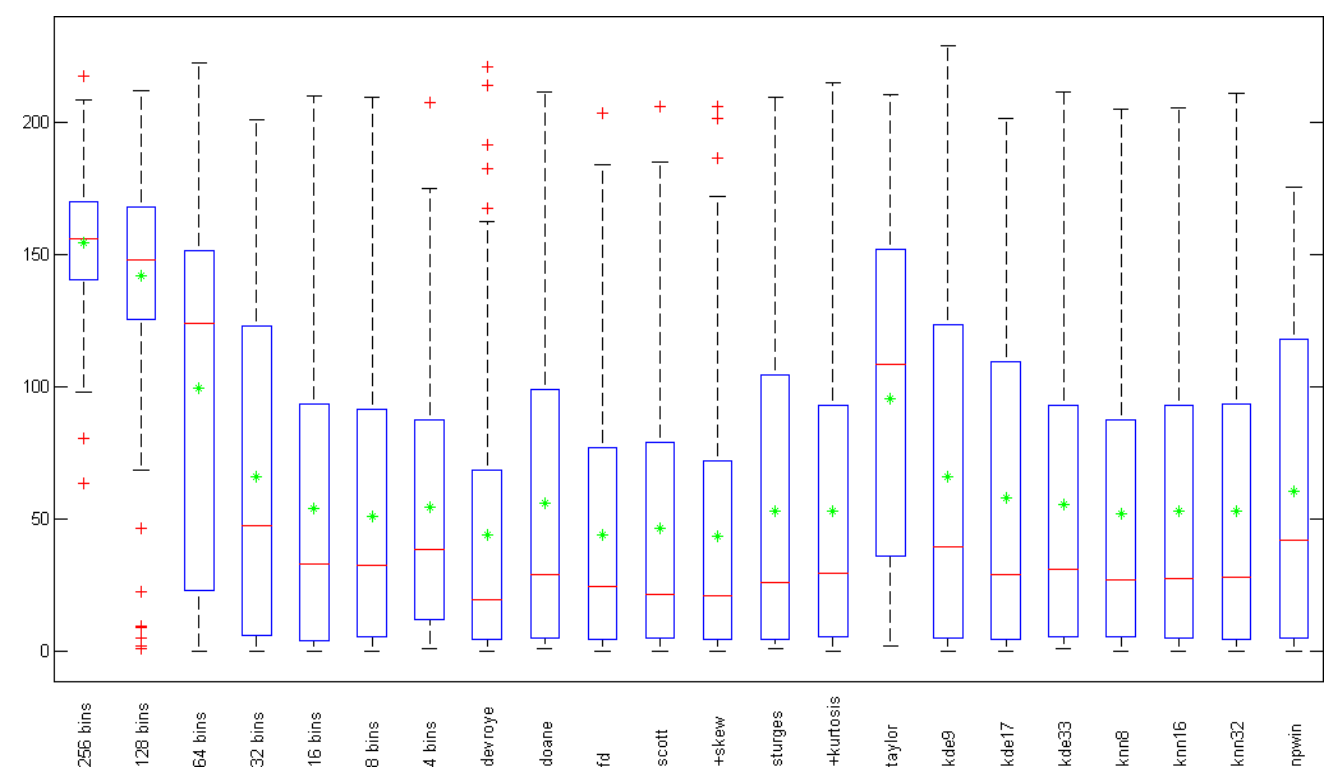

Figure 3: Boxplot of $R e g_{\text {err }}$ for MI simplex registration. Bounding box defines the interquartile range, the star defines the mean and the bar across defines the median. Whiskers define the range and the cross defines any outliers.

number of bins through experimental testing. Of the fixed bin methods, it can be seen that 8 bins gives the lowest registration error when using Simplex and 16 bins gives the lowest registration error when using Simulated Annealing. However, in both cases, using fewer bins than this meant that the registration error increased. This shows then that by using too few bins will degrade registration since salient features in the image are being lost.

When using statistical bin size methods the registration error is reduced further. From these results there are four methods that perform consistently better than fixed bin methods and the advanced probably estimation methods: Devroye's, Freedman-Diaconis', Scott's and Scott's Rule with the skewness factor. The only method that seems to perform poorly in relation to the others is that of Taylor and Kanazawa, giving a result similar to using 64 fixed bins. For the four methods that perform best, it can be seen that Scott's Rule combined with the skewness factor gives the lowest translation error $T$, whilst Scott's Rule gives the lowest rotation error $R$. If we consider only the registration error as given by $R e g_{\text {err }}$, it is Scott's Rule with the skewness factor using the simplex search that achieves the lowest overall reg- 

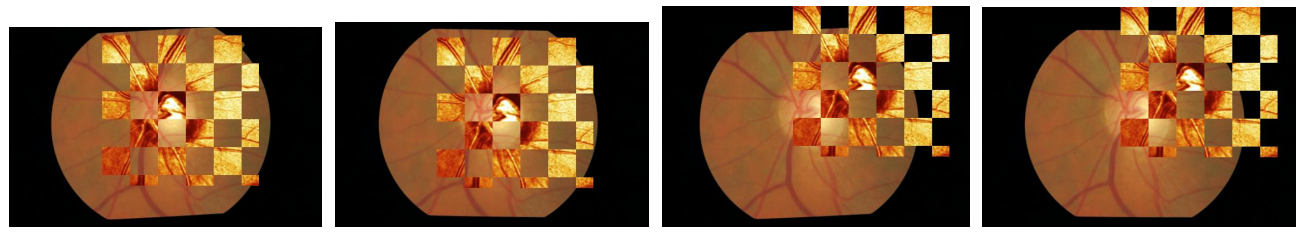

Figure 4: MI registration using four different binning methods. (a) Scott's Rule, (b) 4 bins, (c) 8 bins, (d) 16 bins. Of all methods, only (a) aligns the images correctly.

istration error. These results clearly suggest that statistical bin size selection helps to improve the result for MI registration. Figure 3 shows this trend using a box plot. The mean registration error $R_{e} g_{\text {err }}$ can be plotted for each method allowing us to visualize the results in a clear and concise manner.

Figure 4 shows a registration example using Scott's histogram rule compared to using fixed histogram bin size. In each case where fixed bin size is used, MI fails to successfully register the two images together. The same is also true for when larger fixed bin sizes were used (32, 64, 128 and 256 bins). However, we see that when Scott's rule is used for adaptive bin size selection, the two images are aligned correctly. This highlights the important fact that careful bin size selection $i s$ required and that it is not simply just a case of selecting a low number of bins as has previously been suggested $[14,18,15,16]$.

\subsubsection{Overview of remaining testing scenarios}

Tables 3 and 4 give an overview of registration error for our remaining testing scenarios: NMI using an image pyramid, MI with no pyramid and NMI with no pyramid. The complete set of results can be found in [42].

A similar trend is apparent when using NMI as was seen with MI. In most cases it was seen that using adaptive histogramming techniques improved the result over that of fixed bin sizes, and 256 bins was always found to give the worst performance. From the table, we find that using Scott's Rule combined with simulated annealing and no image pyramid gave the lowest registration error. Compared against the same experiment when using simplex search, the registration error is much greater. Here, it is the search strategy that has failed to find where the similarity measure is maximized rather than the similarity measure not giving the correct result. If the search space consists of many local maxima then it is quite possible that the search algorithm may not find the maximum solution. This highlights the important issue of minimizing the presence of local maxima that the similarity measure generated 


\begin{tabular}{|c|c|c|c|c|c|c|}
\hline \multirow{2}{*}{ Method } & \multicolumn{2}{|c|}{ Mean } & \multicolumn{2}{|c|}{ Median } & \multirow[t]{2}{*}{ Regerr } & \multirow[t]{2}{*}{ Runtime } \\
\hline & $T$ & $\bar{R}$ & $T$ & $\bar{R}$ & & \\
\hline \multicolumn{7}{|c|}{ NMI_Pyramid } \\
\hline 256 bins & 159.2 & 2.9 & 159.8 & 2.5 & 158.9 & 2.69 \\
\hline 32 bins & 56.1 & 2.1 & 27.7 & 1.5 & 57.2 & 2.16 \\
\hline 16 bins & 48.8 & 2.1 & 24.3 & 1.5 & 49.9 & 2.14 \\
\hline 8 bins & 42.5 & 1.8 & 19.0 & 1.5 & 43.5 & 2.15 \\
\hline 4 bins & 51.9 & 2.2 & 37.0 & 2.0 & 53.3 & 2.16 \\
\hline devroye & 48.1 & 1.9 & 23.0 & 1.5 & 49.2 & 2.63 \\
\hline$f d$ & 48.4 & 2.0 & 31.3 & 1.5 & 49.5 & 3.72 \\
\hline scott & 50.8 & 1.9 & 20.0 & 1.5 & 52.0 & 2.62 \\
\hline kde (9) & 58.3 & 2.2 & 28.1 & 2.0 & 59.5 & 4.48 \\
\hline kde (17) & 52.4 & 2.0 & 25.1 & 1.5 & 53.5 & 5.11 \\
\hline$k d e(33)$ & 52.9 & 2.1 & 28.3 & 1.5 & 54.1 & 6.17 \\
\hline \multicolumn{7}{|c|}{ MI_NoPyramid } \\
\hline 256 bins & $\overline{94.3}$ & 2.1 & 114.5 & 1.5 & 94.7 & 10.33 \\
\hline 32 bins & 50.2 & 1.8 & 26.1 & 1.5 & 51.1 & 9.01 \\
\hline 16 bins & 50.6 & 1.9 & 25.8 & 1.5 & 51.6 & 8.99 \\
\hline 8 bins & 49.0 & 1.9 & 27.0 & 1.5 & 50.1 & 8.99 \\
\hline 4 bins & 52.9 & 2.3 & 35.7 & 2.0 & 54.2 & 8.97 \\
\hline$f d$ & 43.2 & 1.7 & 17.1 & 1.0 & 44.2 & 21.33 \\
\hline scott & 43.6 & 1.8 & 16.1 & 1.5 & 44.7 & 11.99 \\
\hline knn (16) & 49.9 & 1.7 & 23.3 & 1.0 & 50.9 & 617.17 \\
\hline knn (32) & 49.9 & 1.5 & 23.3 & 1.0 & 50.8 & 752.39 \\
\hline \multicolumn{7}{|c|}{ NMI_NoPyramid } \\
\hline 32 bins & 48.7 & 1.8 & 19.6 & 1.5 & 49.7 & 8.88 \\
\hline 16 bins & 48.6 & 1.7 & 19.2 & 1.5 & 49.7 & 8.89 \\
\hline 8 bins & 43.9 & 1.7 & 21.9 & 1.0 & 45.1 & 8.86 \\
\hline 4 bins & 49.8 & 2.2 & 32.4 & 1.5 & 51.1 & 8.86 \\
\hline sturges & 45.7 & 1.8 & 13.6 & 1.0 & 46.8 & 9.67 \\
\hline +kurtosis & 45.9 & 1.7 & 14.6 & 1.5 & 47.0 & 20.35 \\
\hline kde (17) & 49.1 & 1.8 & 22.2 & 1.5 & 50.2 & 12.92 \\
\hline
\end{tabular}

Table 3: Registration error results using simplex search for three scenarios: NMI combined with an image pyramid, MI with no pyramid, NMI with no pyramid. (Summary of significant results shown). Translation and $R_{e} g_{e r r}$ are given in pixels, rotation is given in degrees, and runtime is given in seconds.

within the search space. Likewise, it is interesting to observe the difference between when using an image pyramid. It can be seen that registration performs best when no image pyramid is used, however runtime is significantly increased. The issue of local maxima becomes more apparent when the image pyramid is used, to the extent that in some cases the registration fails to recovers at the lower levels of the pyramid. Whilst the improvement to runtime is beneficial, the accuracy of the registration is vital, and so the similarity measure should aim to reduce the occrrence of local maxima.

In terms of runtime, it is clear to see that there is great variation depending on the chosen testing scenario. Using either fixed bins or adaptive bin size, the results in Table 1 are between 2-5 seconds when using simplex search strategy, and between 12-38 seconds when using simulated annealing. When using more advanced methods of probability estimation such as $k \mathrm{NN}$ and NP-windows, the runtime becomes much longer. This becomes even more apparent in our other testing scenarios, where no image pyramid is adopted. The complexity of computing the probability estimation using such an ap- 


\begin{tabular}{|c|c|c|c|c|c|c|}
\hline \multirow[t]{2}{*}{ Method } & \multicolumn{2}{|c|}{ Mean } & \multicolumn{2}{|c|}{ Median } & \multirow[t]{2}{*}{ Regerr } & \multirow[t]{2}{*}{ Runtime } \\
\hline & $T$ & $\bar{R}$ & $T$ & $\bar{R}$ & & \\
\hline \multicolumn{7}{|c|}{ NMI_Pyramid } \\
\hline 256 bins & 146.6 & 2.5 & 148.4 & 2.0 & 146.8 & 24.11 \\
\hline 32 bins & 104.6 & 2.1 & 127.0 & 2.0 & 105.2 & 14.26 \\
\hline 16 bins & 78.4 & 2.1 & 81.3 & 1.5 & 79.1 & 14.39 \\
\hline 8 bins & 75.9 & 2.2 & 83.5 & 1.5 & 77.2 & 14.37 \\
\hline 4 bins & 87.6 & 2.4 & 91.2 & 2.0 & 88.3 & 14.16 \\
\hline devroye & 91.7 & 2.1 & 108.2 & 1.5 & 92.2 & 19.53 \\
\hline$f d$ & 65.8 & 1.9 & 49.3 & 1.5 & 66.4 & 33.65 \\
\hline scott & 83.9 & 2.1 & 90.7 & 1.5 & 84.4 & 19.61 \\
\hline kde (9) & 105.8 & 2.3 & 130.4 & 2.0 & 106.5 & 42.46 \\
\hline$k d e(17)$ & 90.9 & 2.1 & 106.2 & 1.5 & 91.4 & 49.59 \\
\hline kde (33) & 84.6 & 2.2 & 100.1 & 2.0 & 85.5 & 65.20 \\
\hline \multicolumn{7}{|c|}{ MI_NoPyramid } \\
\hline 256 bins & 58.3 & 1.8 & 47.5 & 1.5 & $\overline{59.1}$ & $\overline{70.93}$ \\
\hline 32 bins & 35.3 & 1.7 & 6.1 & 1.0 & 36.5 & 54.54 \\
\hline 16 bins & 35.1 & 1.7 & 6.3 & 1.0 & 36.3 & 57.39 \\
\hline 8 bins & 43.2 & 1.9 & 18.1 & 1.5 & 44.4 & 57.81 \\
\hline 4 bins & 52.4 & 2.1 & 46.1 & 1.5 & 53.4 & 58.64 \\
\hline$f d$ & 28.1 & 1.6 & 5.4 & 1.0 & 29.1 & 152.99 \\
\hline scott & 26.3 & 1.5 & 5.4 & 1.0 & 27.5 & 76.29 \\
\hline knn (16) & 27.2 & 1.5 & 6.1 & 1.0 & 28.9 & 4983.70 \\
\hline knn (32) & 27.5 & 1.6 & 6.1 & 1.0 & 29.4 & 5217.90 \\
\hline \multicolumn{7}{|c|}{ NMI_NoPyramid } \\
\hline 32 bins & 34.7 & 1.6 & 5.7 & 1.0 & 35.7 & 63.84 \\
\hline 16 bins & 37.2 & 1.6 & 6.7 & 1.0 & 38.1 & 60.30 \\
\hline 8 bins & 40.0 & 1.6 & 12.4 & 1.0 & 41.1 & 60.04 \\
\hline 4 bins & 51.1 & 2.1 & 39.7 & 2.0 & 52.2 & 60.11 \\
\hline sturges & 35.8 & 1.6 & 6.3 & 1.0 & 36.8 & 65.33 \\
\hline+ kurtosis & 30.6 & 1.6 & 5.1 & 1.0 & 31.8 & 175.54 \\
\hline kde (17) & 37.9 & 1.9 & 7.6 & 1.0 & 39.0 & 100.71 \\
\hline
\end{tabular}

Table 4: Registration error results using simulated annealing for three scenarios: NMI combined with an image pyramid, MI with no pyramid, NMI with no pyramid. (Summary of significant results shown). Translation and $R_{e} g_{e r r}$ are given in pixels, rotation is given in degrees, and runtime is given in seconds.

proach means that it is not suitable for use in a registration scheme. Of course, as we have already discussed, the methods with low runtime may not necessarily provide the correct registration, and so there is a trade-off between runtime and accuracy. Computing MI using Scott's rule with no pyramid, and searching with simulated annealing we achieve a runtime of 76.29 seconds. This runtime is reasonably acceptable and also delivers the greatest registration accuracy.

\section{Discussion}

In this study we have explored the effects of probability estimation as part of the MI similarity measure. Since MI is based upon the entropy calculation of the probability distribution, how the distribution is computed can play a crucial role in the registration accuracy. Typically, when constructing a histogram for an image, many people would assume to use 256 bins so that each intensity is binned independently. Certainly in the case of MI, we can see this approach gives very poor results. Some studies suggest using a lower 


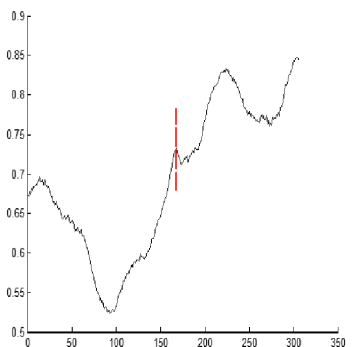

(a)

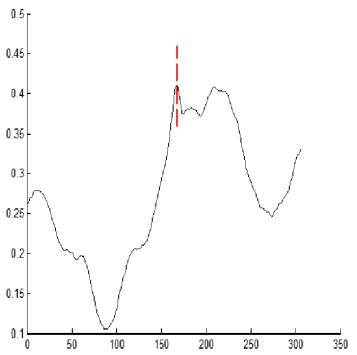

(d)

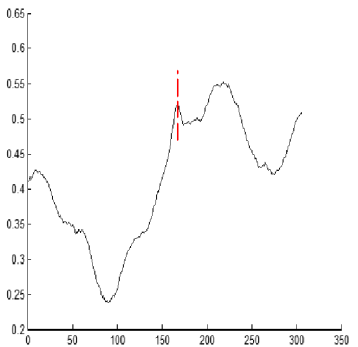

(b)

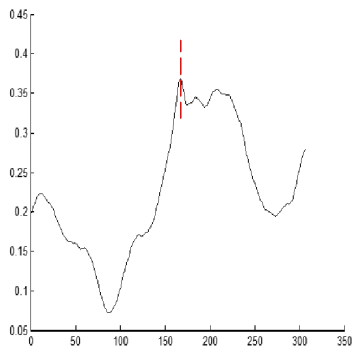

(e)

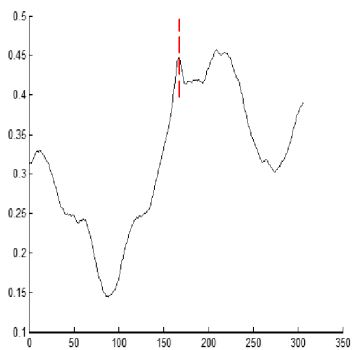

(c)

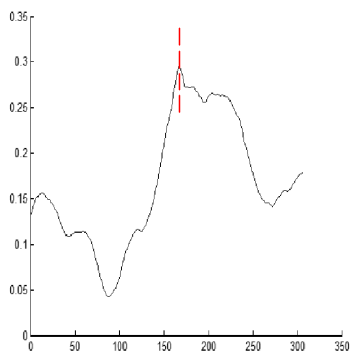

(f)

Figure 5: Surface plots showing MI (y-axis) vs. X-Translation (x-axis) using fixed bin sizes. (a) 256, (b) 128, (c) 64, (d) 32 (e) 16 and (f) 8 bins. The red line indicates the point that should be maximized by MI in each plot.

number of fixed bins [17], which we observe in our results can improve registration. We have incorporated adaptive bin size techniques from statistics literature within registration, and found Scott's Rule, Scott's Rule with the skewness factor, Devroye's Rule and Freedman-Diaconis' Rule, to give the most accurate registration.

We have also compared the statistical bin size approach to other probability estimation methods; Kernel density estimation (KDE), k-Nearest Neighbours (k-NN) and NP-windows, and include these in our testing strategy. The results show that there is no significant improvement to registration accuracy when using these methods compared to the simple histogram, however they take longer to compute. Finally, we have considered the impact that the search optimization scheme can have on registration and also the effects of incorporating an image pyramid as part of the registration search process.

It is surprising to see that 256 bins gives such weak results in comparison to the other methods and so we investigate this further. Figure 5 shows the 
MI results when registering a pair of retinal images (Figure 1), computed only for the X-translation, using different bin sizes. Reducing the parameter space allows us to clearly visualize the difference in the similarity measure. The expected point of registration is given by the dashed vertical line. Using 256 bins, the true registration is a local maximum but not the global maximum. As the bin size is reduced, the true registration becomes more prominent. Even when the true registration is the global maximum (32, 16 or 8 bins), there are other local maxima that could impact on the search strategy. It has been discussed previously that a sparsely-populated histogram will give poor estimation of entropy [43]. By using statistical methods, we can now determine a suitable number of bins rather than simply choosing an arbitrary number of bins in the hope that it performs well.

Interestingly, in some particularly challenging cases, we found that the failure of registration to be as a result of weaknesses in the MI algorithm. Figure 6 shows an example of this from our retinal image dataset. In 6(a), MI fails to register the two images. However, the MI score is actually maximized, giving a better result than the ground truth registration 6(b). This result is noticeable for this particular example for all probability estimation methods covered in this study. This highlights that whilst statistical probability estimation may improve upon traditional techniques, MI is still not a fully reliable similarity measure. Kubecka et al. [44] also studied the registration of these two image modalities using MI, and whilst they did not consider the affect of bin size estimation, they did conclude that "the mutual information had global extreme out off the point of subjective registration". They do not give quantitative results for using MI. It is recognized that MI lacks spatial information [6], which if this was incorporated into the algorithm could help to improve registration performance. Some more recent methods consider this (e.g., [45, 46, 47, 48]), however they are more computational-intensive than MI and have significantly longer runtime requirements.

\section{Conclusion}

We have investigated the Mutual Information registration algorithm and highlighted the importance of probability density estimation as part of the algorithm. In constructing the histogram for probability estimation, we show that careful bin size selection does have a direct impact on the registration result. Many previous studies using MI omit this detail or do not truly consider how this could affect the accuracy of registration. We have con- 


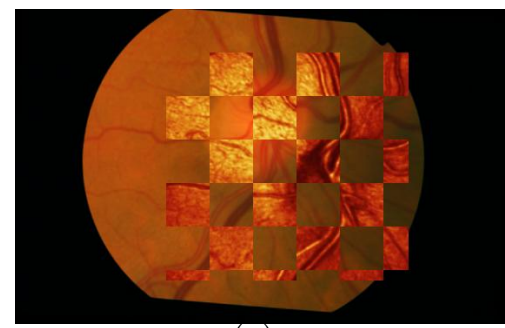

(a)

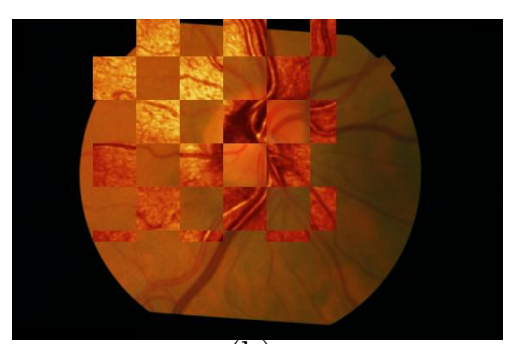

(b)

Figure 6: An example of difficult registration. (a) Registration given by maximum MI. (b) Ground truth registration.

ducted a comprehensive empirical study for performing image registration that demonstrates the impact of adaptive bin size selection methods, as well as other registration parameters such as search optimization strategies and inclusion of an image pyramid. We show that this approach can improve upon fixed bin size methods, and significantly reduces the need for extensive experimental testing to find a suitable number of bins $[14,13,15,16]$.

Previously, many MI registration applications have focused on common modalities such as MR and CT imaging without much concern regarding bin size, and have enjoyed great success. As more advanced imaging technologies are introduced though, traditional MI registration may not suffice for accurate results. We have seen that this is the case for multi-modal retinal images, and that registration parameters needs to be considered more carefully for challenging modalities. Likewise, we would expect that other modalities such as $\mathrm{PET} / \mathrm{CT}$ registration would also benefit from adaptive bin size selection. More recently, MI has received criticism for not including spatial information, and so new algorithms have been proposed to tackle this. However, MI remains as a simple and reliable similarity measure that can be easily implemented and is fast to compute compared to more modern methods. In addition, the use of adaptive histogram bin size selection methods can yield significant improvements on the traditional MI algorithm, meaning that it should not be neglected for modern registration tasks.

\section{References}

[1] P. A. Viola and W. M. Wells III. Alignment by maximization of mutual information. In $I C C V$, pages 16-23, 1995. 
[2] A. Collignon, F. Maes, D. Delaere, D. Vandermeulen, P. Suetens, and G. Marchal. Automated multimodality medical image registration using information theory. In Proc. 14th Int. Conf. Information Processing in Medical Imaging; Computational Imaging and Vision 3, pages 263-274, 1995.

[3] C. Studholme, D. L. G. Hill, and D. J. Hawkes. An overlap invariant entropy measure of 3D medical image alignment. Pattern Recognition, 32(1):71-86, 1999.

[4] C. E. Shannon. A mathematical theory of communication. Bell System Technical Journal, 27:379-423, 623-656, 1948.

[5] C. Y. Mardin, F. K. Horn, J. B. Jonas, and W. M. Budde. Preperimetrix glaucoma diagnosis by confocal scanning laser tomography of the optic disc. British Journal of Ophthalmology, 83:299-304, 1999.

[6] J. P. W. Pluim, J. B. Antoine Maintz, and M. A. Viergever. Mutual information based registration of medical images: A survey. IEEE Trans. Med. Imaging, 22(8):986-1004, 2003.

[7] J. Beirlant, E. J. Dudewicz, L. Györfi, and E. C. Meulen. Nonparametric entropy estimation: An overview. International Journal of the Mathematical Statistics Sciences, 6:17-39, 1997.

[8] L. Paninski. Estimation of entropy and mutual information. Neural Computation, 15:1191-1254, 2003.

[9] G. Egnal. Image registration using mutual information. Technical report, University of Pennsylvania, 1999.

[10] L. Birgé and Y. Rozenholc. How many bins should be put in a regular histogram. Technical report, Université Paris VI, UMR CNRS 7599, Université du Maine, 2002.

[11] F. Maes, A. Collignon, D. Vandermeulen, G. Marchal, and P. Suetens. Multimodality image registration by maximization of mutual information. IEEE Trans. Med. Imaging, 16(2):187-198, 1997.

[12] N. D. H. Dowson and R. Bowden. A unifying framework for mutual information methods for use in non-linear optimisation. In $E C C V$ (1), pages 365-378, 2006. 
[13] R. Lachner. From Nano to Space. Springer Berlin Heidelberg, 2008.

[14] N. Ritter, R. A. Owens, J. R. Cooper, R. H. Eikelboom, and P. P. Van Saarloos. Registration of stereo and temporal images of the retina. IEEE Trans. Med. Imaging, 18(5):404-418, 1999.

[15] H. Nam, R. A. Renaut, K. Chen, H.Guo, and G. E. Farin. Improved inter-modality image registration using normalized mutual information with coarse-binned histograms. Communications in Numerical Medthods in Engineering, 25:583-595, 2009.

[16] J. Kang, C. Xiao, M. Deng, J. Yu, and H. Liu. Image registration based on harris corner and mutual information. In Electronic and Mechanical Engineering and Information Technology (EMEIT), 2011 International Conference on, volume 7, pages 3434 -3437, aug. 2011.

[17] Y. Zhu and S. M. Cochoff. Influence of implementation parameters on registration of MR and SPECT brain images by maximization of mutual information. Journal of Nuclear Medicine, 43(2):160-166, 2002.

[18] J. Tsao. Interpolation artifacts in multimodality image registration based on maximization of mutual information. IEEE Trans. Med. Imaging, 22(7):854-864, July 2003.

[19] P. L. Davies, U. Gather, D. Nordman, and H. Weinert. Constructing a regular histogram - a comparison of methods. Technical report, Technical University Eindhoven, 1997.

[20] H. A. Sturges. The choice of a class interval. American Statistical Association, pages 65-66, 1926.

[21] D. W. Scott. On optimal and data-based histograms. Biometrika, 66(3):605-610, 1979.

[22] R. J. Hyndman. The problem with Sturges' rule for constructing histograms. Technical report, Melbourne University, Australia, 1995.

[23] D. Freedman and P. Diaconis. On the histogram as a density estimator. Zeitschrift fur Wahrscheinlichkeitstheorie und verwandte Gebiete, 57(4):453-476, 1981. 
[24] L. Devroye and L. Györfi. Nonparametric density estimation: The $L_{1}$ view. Journal of the Royal Statistical Society. Series A (General)., 148(4):392-393, 1985.

[25] C. C. Taylor. Akaike's information criterion and the histogram. Biometrika, 74:636-639, 1987.

[26] D. P. Doane. Aesthetic frequency classifications. The American Statistician, 30(4):181-183, November 1976.

[27] D. W. Scott. Multivariate density estimation: theory, practice and visualization. John Wiley and Sons, 1992.

[28] J. D. Wichard, R. Kuhne, and A. ter Laak. Binding site detection via mutual information. Proc. of the IEEE World Congress on Computational Intelligence, pages 1770-1776, June 2008.

[29] Hideaki Shimazaki and Shigeru Shinomoto. A method for selecting the bin size of a time histogram. Neural Comput., 19(6):1503-1527, June 2007.

[30] Y. Kanazawa. Hellinger distance and akaike's information criterion for the histogram. Statistics \& Probability Letters, 17:293-298, 1993.

[31] A. J. Izenman. Recent developments in nonparametric density estimation. Journal of the American Statistical Association, 86(413):205-224, 1991.

[32] H. Xie, L. E. Pierce, and F.T. Ulaby. Mutual information based registration of SAR images. Geoscience and Remote Sensing Symposium, IGARSS '03. Proc. IEEE International, 6:4028-4031, July 2003.

[33] N. Kwak and C. Choi. Input feature selection by mutual information based on Parzen Window. IEEE Transactions on Pattern Analysis and Machine Intelligence, 24(12):1667-1671, 2002.

[34] C. O. Daub, R. Steuer, J. Selbig, and S. Kloska. Estimating mutual information using b-spline functions - an improved similarity measure for analysing gene expression data. BMC Bioinformatics, 5:118, 2004.

[35] A. Kraskov, H. Stögbauer, and P. Grassberger. Estimating mutual information. Physical Review E, 69(6):066138, June 2004. 
[36] N. Nicolaou and S. J. Nasuto. Mutual information for EEG analysis. Proc. 4th IEEE EMBSS UKRI Postgraduate Conference on Biomedical Engineering and Medical Physics (PGBIOMED'05), pages 23-24, 2005.

[37] N. D. H. Dowson, R. Bowden, and T. Kadir. Image template matching using mutual information and NP-windows. In ICPR (2), pages 11861191, 2006.

[38] A. Rajwade, A. Banerjee, and A. Rangarajan. Continuous image representations avoid the histogram binning problem in mutual information based image registration. In ISBI, pages 840-843, 2006.

[39] Heidelberg Engineering, Heidelberg, Germany. Quantitative Threedimensional Imaging of the Posterior Segment with the Heidelberg Retina Tomograph, 1999.

[40] W. K. Pratt. Digital Image Processing. Wiley, New York, 2 edition, 1991.

[41] J. Vandekerckhove. General simulated annealing algorithm (http://www . mathworks.com/matlabcentral/fileexchange/10548), MATLAB central file exchange, June 2008.

[42] P. A. Legg. Multimodal retinal imaging: Improving accuracy and efficiency of image registration using Mutual Information. $\mathrm{PhD}$ thesis, School of Computer Science and Informatics, Cardiff University, 2010.

[43] L. Paninski. Estimating entropy on $m$ bins given fewer than $m$ samples. IEEE Trans. on Information Theory, 50(9):2200-2203, September 2004.

[44] L. Kubecka, M. Skokan, and J. Jan. Registration of bimodal retinal images - improving modifications. Engineering in Medicine and Biology Society, 2003. IEMBS '03. 25th Annual International Conference of the IEEE, 1:599-602, September 2003.

[45] P. A. Legg, P. L. Rosin, D. Marshall, and J. E. Morgan. A robust solution to multi-modal image registration by combining mutual information with multi-scale derivatives. In MICCAI, volume 1, pages 616-623, 2009.

[46] J. P. W. Pluim, J. B. Antoine Maintz, and M. A. Viergever. Image registration by maximization of combined mutual information and gradient information. IEEE Trans. Med. Imaging, 19(8):809-814, 2000. 
[47] D. Rueckert, M. J. Clarkson, D. L. G. Hill, and D. J. Hawkes. Non-rigid registration using higher-order mutual information. Medical Imaging: Image Processing, pages 438-447, 2000.

[48] D. B. Russakoff, C. Tomasi, T. Rohlfing, and C. R. Maurer Jr. Image similarity using mutual information of regions. In $E C C V$ (3), pages 596-607, 2004. 